\title{
The pre-diagnostic trajectory
}

There are many pathways and trajectories that patients take on their way to seeking medical attention and to a final diagnosis. The journeys may start with the symptoms themselves or with prompts from the environment around them. This information that motivates people to seek medical attention may come from the ubiquitous modern media, or from online information or misinformation. Prompts to seek healthcare may also come from the illness experiences of relatives, friends or colleagues.

While on the way to see my own doctor I carefully go through, in my mind, what I am going to say to her and usually have already terrified myself with the diagnostic possibilities of my symptoms. On arrival we talk about our mutual social lives (called phatic communion) and then proceed to the symptoms themselves, but by this time I have lost the thread of my neatly ordered history. I begin in the middle and embellish a mixture of patchy stories with added digressions on what I believe are the causes of my symptoms.

During this process, it is difficult for her to separate the wheat from my chaff, but this is often the process that we have to go through with our patients. They now arrive with already halfformed ideas of their diagnoses, which they have received from the morning radio talk show.

Self-diagnosis or diagnosis by a doctor does not necessarily occur as a once-off event (called a diagnostic moment), but is often an accumulative process. There is firstly a stirring in the mind which is then followed by a pathway of consultations and investigations that eventually ends in the diagnosis (called the pre-diagnostic trajectory). In many cases these days, the initial stimulus may not be made by the symptoms themselves, but may be started by prompts or cues from information received from outside the patient. These are the inchoate, semi-formed thoughts that often arise following health screening or education programs.

In this lead-up, we start to assemble evidence in our minds (called diagnostic assemblage) which sets our thoughts in order (or in my case out of order). This then initiates our health seeking behaviour. There are also those chance pieces of information that we pick up as "snippets" from overheard conversations or from passing media programs.

What follows is the trajectory to seek help from a significant family member, respected acquaintance, the pharmacist or medical practitioner. The pathway often includes an alternative or traditional healer where a culturally-based causation may be added as well.

We can actually jog along for quite some time either denying or suppressing the possibilities of various diagnoses until an event or additional information is received, which kick-starts the process of proactively seeking a diagnostic label.
When I started practice in the late 1960s my patients were diagnostically naïve with very limited knowledge of the functions of the body and its ailments. I have watched over the years as education has improved and now my patients are mostly well-informed. By the time they see me they may "know" their clinical diagnoses (or misdiagnoses) although these may not be fully articulated or formulated in their minds. I have the impression that most of them do not inform me of these prior labels or names that they have formed but wait to see whether I will confirm them or propose alternatives.

At this stage I find it useful to ask the patient what they believe are the causes of their symptoms. The preconceptions can then be laid on the table and either followed up or other avenues can be negotiated. The patient may make several subtle offers during their presentations and these can then be taken up and discussed. Suspicions and anxieties can often be allayed at this early stage. During this journey there is a continual heuristic process of either acceptance or denial and some bargaining with one's own health, religious and cultural beliefs.

Along the way there may also be "accelerators" to the patient seeking help, such as a telephone call received after a laboratory or imaging test has been done. The patient is alerted to the fact that something may be wrong when requested to attend for a hastened follow-up appointment with an urgency that has not been anticipated. Suspicions may be confirmed at the follow-up consultation by small changes in the normal routines (called breaks in the ceremonial order of the medical consultation). The patient picks up on the non-verbal communication of the doctor's demeanour and the give-aways of over-sympathetic comments by the staff. Even slight deviations in protocol such as the rearrangement of the chairs as well as significant silences are causes of heightened awareness in the patient. Although these are seemingly minor changes, they may be deeply significant to the patient (called the salience of the prosaic). These are the small incidents that may alert the patient to the fact that something is wrong, such as when the ultrasonographer, who has been merrily chatting away, suddenly goes silent.

We now live in a world overloaded with information that is within easy access of our patients. If I have time, I try to briefly ask about the routes and trajectories that the patients have made before seeing me. It often expedites the outcomes as well as allaying their diagnostic anxieties and uncertainties.

Chris Ellis is a family physician in Pietermaritzburg, KwaZulu-Natal

E mail: cristobalellis@gmail.com 